https://doi.org/10.48009/2_iis_2006_339-343

\title{
AN EXPLORATORY INVESTIGATION OF INFORMATION TECHNOLOGY ETHICS FACTORS
}

\author{
Alan R. Peslak, Penn State University, arp14@psu.edu
}

\begin{abstract}
Information technology (IT) has proliferated at an unprecedented rate in our society. Technical advances have come quickly yet the social and ethical infrastructure to support these advances has been slow in development. A group of individuals have developed a general code of ethical conduct for information technology professionals to begin to identify the proper ethical choices that IT professionals should make. This paper examines this code and explores whether there are underlying ethical constructs that underlie the code. After examining ten key provisions of the code, it was found through exploratory factor analysis that there were two factors which emerged-information technology integrity and information technology security. There were found to be no differences in level of agreement with these IT ethical factors based on several demographic variables. The implications of these finding for educators, researchers and practitioners are briefly discussed.
\end{abstract}

Keywords: Information Technology (IT), Ethics, IT and Ethics, Code of Ethics, Ethical Factors, IT Security, IT Integrity

\section{INTRODUCTION AND LITERATURE REVIEW}

Rapid technological advances have not been accompanied by progress on related information technology ethical issues. Though our information systems have evolved from data processing, through information systems, to artificial intelligence and embedded systems, there has not been sufficient advancement in dealing with the ethical issues these technologies have spawned [1]. Ogburn's cultural lag theory suggests that technology is advancing faster than the ethical infrastructure necessary to control and support these gains [8]. It is therefore vital to expand the ethical research currently being performed in information technology to help bridge the gap between behavior and information science advances. Understanding unethical IT behavior can have tremendous returns. There are many recent examples of the impact of unethical IT behavior including problems such as software piracy, virus development and illegal access, estimated at costing corporations losses of billions of dollars per year [11]. There are studies that have begun to explore the unique issues associated with codes of conduct and information technology ethics. Many ethical issues have been identified as emerging from the tremendous growth of electronic commerce. These include security of online information, Internet privacy, cybersquatting, online marketing to children and online conflicts of interest [13].

One of the ways of addressing professional ethics is thru the use of Codes of Ethics. The medical, legal, and accounting professions all have long established self-regulatory organizations and have developed specific codes of ethics and conduct to deal with moral issues that arise within their areas. The success of these codes has come under some scrutiny in recent years with scandals such as Enron, but overall these codes have provided some measure of success in dealing with ethical dilemmas. The information technology profession does not currently have such a strong self-regulatory organization, but there are some groups that have developed Codes of Ethics. One of the most widely recognized and publicized is the ACM (Association for Computing Machinery) Code of Ethics and Professional Conduct enacted in 1992 [2]

The preamble of the code includes the following:

Commitment to ethical professional conduct is expected of every member (voting members, associate members, and student members) of the Association for Computing Machinery (ACM).

The Code and its supplemented Guidelines are intended to serve as a basis for ethical decision making in the conduct of professional work. Secondarily, they may serve as a basis for judging the merit of a formal complaint pertaining to violation of professional ethical standards. [2]

This Code is the most comprehensive guideline to bridge the gap between information technology and ethics. Its content is studied in this manuscript.

Mere existence of Codes of conduct has been found to have no impact on ethical decision making $[4,6]$. 
Culture has been found to be more important than codes on influencing behavior [12]. However, it has been found that IT ethics can be successfully taught [5]. Vitell and Davis [14] found that ethics can improve "when top management makes it clear that ethical behavior will be rewarded and unethical behavior will be punished." Hoff [7] suggests that individuals can be trained in ethical decision making in order to make proper ethical decisions. Therefore, exploring the nature of IT ethics and determining both its underlying elements as well as its demographic variability are productive efforts. Understanding these issues can lead to better programs to foster ethical education and improve ethical behavior.

\section{RESEARCH METHODOLOGY}

This study had several research objectives. First, it was an attempt to uncover via exploratory factor analysis (EFA) underlying factors for similar and common information technology issues as expressed in the ACM Code of Ethics. The use of EFA in finding underlying common factors is well established in information systems research [9].

H1: There will be underlying ethical factors within common IT ethical issues.

The second objective was to determine whether there were significant differences in the way demographic groups viewed the underlying ethical factors. The specific hypotheses were as follows.

H2: Gender will not play a significant role in the way major information technology factors are viewed.

Gender has been found to have no significant impact on information technology ethics [3].

Two other demographic variables are studied: age and ACM membership.

H3: Age will not play a significant role in the way major information technology factors are viewed.

H4: ACM membership will not play a significant role in the way major information technology factors are viewed.

In order to test these hypotheses, an online survey was prepared including 10 specific ethical imperatives extracted from the overall ACM Code of Conduct. The extracted items are included in the Results Table 1. They were selected by the author and represent many of the significant current information technology ethical issues. The survey asked the level of agreement with the ethical imperatives on a five point scale from strongly disagree to strongly agree. This report is part of a broader study examining different aspects of IT ethics and the ACM Code of Conduct. Participants were IT students in a small Northeastern US university as well as faculty and professionals who belong to a University technology listserv. The use of students as a subject group is well established in IT ethics research [3]. Participation ranged from $100 \%$ in the author's class to $8 \%$ from the listserv. In total there were between 120 and 125 usable responses to the survey based on the questions (not all questions were answered by all).

All results were analyzed using SPSS 10.0. Specifically to address the first hypothesis, exploratory factor analysis was performed along with scale reliability testing. Two measures of variance were used to test hypotheses two to four.

\section{RESULTS}

H1: There will be underlying ethical factors within common IT ethical issues.

The results of the exploratory factor analysis found two distinct factors emerging from the code, utilizing eight of the ten code statements. Two statements were not found to be related to other variables. The EFA was conducted using Varimax rotation and the statements and the results are shown in Table 1. The results clearly show two factors emerging. (An Eigenvalue of 1.0 was used similar to Moore [9]). A review of the statements associated with these common factors suggests that they deal with two common areas IT Integrity and IT Security. The component elements are highlighted in bold in the table. The total variance explained by these factors represents approximately 61 percent. 
Table 1. Rotated Component Matrix

\begin{tabular}{|c|c|c|c|}
\hline & & Component & \\
\hline & & 1 & 2 \\
\hline False & $\begin{array}{l}\text { (You should) not make deliberately false or deceptive claims about a } \\
\text { system or system design, but ... instead provide full disclosure of all } \\
\text { pertinent system limitations and problems. }\end{array}$ & .754 & $8.950 \mathrm{E}-02$ \\
\hline Copy & Unauthorized duplication of materials must not be condoned. & .732 & .171 \\
\hline Privacy & $\begin{array}{l}\text { It is the responsibility of professionals to maintain the privacy and } \\
\text { integrity of data describing individuals }\end{array}$ & .200 & .830 \\
\hline Confidential & $\begin{array}{l}\text { Respect all obligations of confidentiality to employers, clients, and } \\
\text { users unless discharged from such obligations by requirements of the } \\
\text { law or other principles of this Code. }\end{array}$ & .126 & .863 \\
\hline Quality & $\begin{array}{l}\text { Strive to achieve the highest quality, effectiveness and dignity in both } \\
\text { the process and products of professional work. }\end{array}$ & .654 & .298 \\
\hline Risk & $\begin{array}{l}\text { Give comprehensive and thorough evaluations of computer systems } \\
\text { and their impacts, including analysis of possible risks. }\end{array}$ & .657 & .393 \\
\hline Resources & $\begin{array}{l}\text { Access computing and communication resources only when } \\
\text { authorized to do so. }\end{array}$ & .425 & .590 \\
\hline Users & $\begin{array}{l}\text { Ensure that users and those who will be affected by a system have } \\
\text { their needs clearly articulated during the assessment and design of } \\
\text { requirement }\end{array}$ & .719 & .171 \\
\hline
\end{tabular}

Extraction Method: Principal Component Analysis. Rotation Method: Varimax with Kaiser Normalization.

a Rotation converged in 3 iterations.

Factor 1 includes statements related to false claims, not copying materials, quality of work, full risk articulation, and inclusion of user needs. All the statements deal with issues of personal integrity:

(You should) not make deliberately false or deceptive claims about a system or system design, but ... instead provide full disclosure of all pertinent system limitations and problems. You should not deceive but give full information. Unauthorized duplication of materials must not be condoned. You should not steal intellectual property. Strive to achieve the highest quality, effectiveness and dignity in both the process and products of professional work. You should do quality work. Give comprehensive and thorough evaluations of computer systems and their impacts, including analysis of possible risks. You should professionally advise your users of all risks. Ensure that users and those who will be affected by a system have their needs clearly articulated during the assessment and design of requirements. You should do your job properly by including all users in the design. These concepts can be summarized as overall professionalism and morality in your actions as an information technology developer or service provider. These concepts suggest an overall concept of IT Integrity. The scale reliability of this factor was performed and Cronbach's alpha was found to be acceptable at .7814. According to Nunnally [10] .70 is the cutoff for acceptable reliability.

Factor 2 includes statements related to overall IT security including privacy, confidentiality, and access to communication resources. All these issues relate directly to security of data and information. It is the responsibility of professionals to maintain the privacy and integrity of data describing individuals. Respect all obligations of confidentiality to employers, clients, and users unless discharged from such obligations by requirements of the law or other principles of this Code. Access computing and communication resources only when authorized to do so. These concepts suggest an underlying concept of IT Security which is found to be a separate and distinct factor from IT Integrity. The scale reliability of this factor was performed and Cronbach's alpha was over the acceptable range at .7079.

Hypothesis 1 was supported. There were found to be underlying ethical factors in the series of varied 
ethical imperatives. There are clearly two different factors that have been uncovered in the analysis. One is personal and deals with individual actions. The other is more abstract and deals with overall IT privacy and confidentiality. The factors are suggested to be IT Integrity and IT Security.

The next series of hypotheses centered on determining whether there were found to be significant differences in levels of agreement with these ethical factors based on demographic variables. In order to test these hypotheses, the factor scores were saved as variables in SPSS and then analysis of variance was performed on the difference in means based on demographic variables. Since the distribution of these variables appeared as though they may not be normal, an additional Kruskal-Wallis test was performed. This is a non-parametric test based on ranks and does not require normalcy for statistical testing accuracy. Both the ANOVA and the Kruskal-Wallis test confirm the null hypotheses that there are no significant differences in levels of agreement with information technology ethical factors based on the common demographics of Gender, Age, and Membership in ACM at $\mathrm{p}<.05$. The summarized results of these tests are shown in Table 2.

Table 2. Tests for Significance

\begin{tabular}{|l|c|c|c|}
\hline Demographic & Factor & ANOVA significance & Kruskal-Wallis significance \\
\hline Gender & IT Integrity & .129 & .155 \\
\hline & IT Security & .158 & .169 \\
\hline Age & IT Integrity & .149 & .074 \\
\hline & IT Security & .180 & .317 \\
\hline ACM Member & IT Integrity & .160 & .126 \\
\hline & IT Security & .158 & .153 \\
\hline
\end{tabular}

All the remaining hypotheses were supported. Gender does not play a significant role in the way major information technology ethical factors are viewed. Age does not play a significant role in the way major information technology ethical factors are viewed. ACM membership does not play a significant role in the way major information technology ethical factors are viewed.

\section{CONCLUSIONS AND IMPLICATIONS}

As with any study, there are limitations that need to be noted. First, the survey only studied students, faculty, staff, and practitioners associated with a small Northeast U.S. University. The results may not be applicable to a wider population. Results need to be confirmed with a broader sample. Secondly, the survey only measured a subset of the overall ACM Code of Ethics. A more complete detailing of provisions of the code may have yielded additional factors. Also, the Code though thorough is not fully current and comprehensive and does not include specific issues such as employee monitoring and instant messaging abuse. Nevertheless, this study has provided interesting findings, furthering the study of information technology ethics.

This exploratory study found that there are two factors which underlie common information technology ethical issues - IT Integrity and IT Security. The views on numerous issues related to these areas are sufficiently similar to combine these responses into common factors. This is an important and useful finding for educators, researchers, and practitioners. The teaching of IT ethics can present unique challenges with rapidly changing technology. The uncovering of two common factors allows education and instruction to be more narrowly focused on the implications of the two uncovered IT factors. This should simplify and enhance IT ethical education. The factors should prove useful for IT researchers as they can now provide a framework for further demographic and causal analysis. There are many fertile areas for further research including using these factors in more detailed IT ethical models, exploring the influence of these factors on ethical behavior, and confirming the results with broader cross sections of society and geography. Practitioners face an increasingly dangerous environment within IT integrity and security. These factors should help in outlining a behavior modification program to improve IT ethics in practice.

The second major conclusion of the study was that neither of the IT ethical factors showed any significant variance based on the demographic variables studied. Neither gender nor age nor membership in the ACM resulted in significant variation in the level of agreement with the IT ethical statements. This again holds interest for educators, researchers, and practitioners. Practitioners and educators do not need to recognize noted demographic differences in preparing their IT ethical programs. IT ethics issues show no gender, age, or 
professional membership bias. Researchers can take this lack of demographic variation as further evidence of a broad recognition of the importance of IT ethics issues.

Overall, the results further the overall exploration of an extremely vital component of information technology today, the ethical use of such technology. Though problems continue, little work has been done to investigate and clarify the underlying issues related to IT ethical issues. In addition, little work has been performed on how these issues vary across demographic groups. This contributes to the development of an ethical body of research and should be further extended by more comprehensive study.

\section{REFERENCES}

1. Abi-Read, M. (1999). Code of ethics? Which one? SIGCSE Bulletin, 31(2), 73-77.

2. Association for Computing Machinery. (1997). ACM Code of Ethics and Professional Conduct. Available online at: http//www.acm.org/constitution/code.html

3. Calluzo V. \& Cante C. (2004). Ethics in information technology and software use. Journal of Business Ethics, 51, 301-312.

4. Cleek, M. \& Leonard, S. (1998). Can corporate codes of ethics influence behavior? Journal of Business Ethics, 17, 619-630.
5. Couger, J. (1989). Preparing IS students to deal with ethical issues. MIS Quarterly, 13(2), 211218.

6. Harrington, S. (1996). The effect of codes of ethics and personal denial of responsibility on computer abuse judgments and intentions. MIS Quarterly, 20(3), 257-278.

7. Hoff, J. (1989). Students, ethics, and surveys. Journal of Business Ethics, 8, 823-825.

8. Marshall, K. (1999). Has technology introduced new ethical problems? Journal of Business Ethics, 19, 81-90.

9. Moore, J. (2000). One road to turnover: An examination of work exhaustion in technology professionals. MIS Quarterly, 24(1), 141-168.

10. Nunnally, J.C. (1978). Psychometric Theory. New York: McGraw-Hill.

11. Peterson, D. (2002). Computer ethics: The influence of guidelines and universal moral beliefs. Information Technology \& People, 15(4), 346-361.

12. Sims, R. \& Brinkmann, J. (2003). Enron ethics (or culture matters more than codes). Journal of Business Ethics, 45, 243-256.

13. Stead, B. \& Gilbert, J. (2001). Ethical issues in electronic commerce. Journal of Business Ethics, 34, 75-85.

14. Vitell. S. \& Davis, D. (1990). Ethical beliefs of MIS professionals: The frequency and opportunity for unethical behavior. Journal of Business Ethics, 9, 63-70. 